\title{
Optimal Joint Rate and Power Allocation in CDMA Networks
}

\author{
R.J. Boucherie ${ }^{1}$, A.I. Endrayanto ${ }^{1}$, and A. F. Gabor ${ }^{2}$ \\ ${ }^{1}$ Faculty of Electrical Engineering, Mathematics and Computer Science, \\ University of Twente, 7500 AE Enschede, The Netherlands \\ ${ }^{2}$ Faculty of Mathematics and Computer Science and EURANDOM, \\ Eindhoven University of Technology, 5600 MB, Eindhoven, The Netherlands
}

\begin{abstract}
In this paper we propose a polynomial time algorithm for the optimal rate and power allocation problem in a two cell CDMA network. We assume continuous rates and limited powers for the base stations.
\end{abstract}

\section{Introduction}

The inherit capacity restrictions due to scarce resources are fundamental problems in the operation of a wireless CDMA system. At the operational level (time scale of minutes), load fluctuations occur due to randomness in call generation and call lengths. At this time scale, load balancing is carried out via power and rate assignment as well as a reconfiguration of calls over cells. Power and rate assignment requires an underlying policy or network optimality criterion.

Common optimality criteria for CDMA network optimization are equal rate to all calls, or maximum total network data rate. Equal rates to all calls seems fair from a call perspective, but is rather inefficient in networks sustaining a normal load, mainly due to calls far away from base transmitter stations (BTS) causing a large amount of interference, and therefore a substantial reduction in network capacity. An important question in achieving maximum data rate is the assignment of data rates to individual calls. This assignment is clearly closely related to power assignment. This paper addresses, in an analytical setting, the joint power and rate assignment in two cells in a CDMA network.

Literature. The joint rate and power assignment problem for CDMA systems has received considerable attention over the last decades. Due to the complexity of the problems, several restrictions have been made, in order to obtain mathematically tractable models.

The most common simplifications are considering a cell in isolation, thus neglecting the interference effects, or assuming some extra properties of rates/ powers, like unlimited rates or powers. For the simplified model of a single cell in isolation, down link power assignment schemes for maximizing the throughput or minimizing the total power in the cell are proposed in 8512. Resource assignment in a multi cell environment is more complex than in one cell, due to the interferences caused by users in adjacent cells. It has been studied in the framework of cell-breathing for fixed data rates, see e.g. the pioneering work of 
[7, 11] that consider the uplink, that in the early days of CDMA was considered to be the bottle-neck. For the down link, joint rate and cell assignment is studied in 9] via a dynamic pricing algorithm under the assumption that each base station maximizes its total system utility, without considering the status of the other cells. In 2] an distributed algorithm for assigning base station transmitter (BTSs) powers such that the common rate of the users is maximized is described. In 10, Perron-Frobenius theory is used to design an approximate algorithm for a model with multiple rates, which permits the use of techniques from convex optimization. In 4], the authors propose a polynomial time approximation scheme for the joint rate and power assignment problem under the assumption that the rates allocated are discrete and the power of the base stations is unlimited.

Contribution and Outline. This paper proposes a fast and exact joint rate and power allocation algorithm in the down link of a telecommunication network formed by two cells, where the base stations transmit at limited powers. Thus, we incorporate in our model two important aspects of a CDMA network, namely interference and limited powers. We assume that the rates are continuous and may be chosen from a given interval. This assumption seems realistic, since in a CDMA system data rates may be rapidly modified in accordance with channel conditions, resulting in an average rate that lies in an interval.

Section 2 provides the model and describes the resulting optimization problem. Due to the impact of the interference between users in different cells, this problem is much more difficult then the rate/power optimization problem in one cell, and it is more difficult then the problem with unlimited powers. In Section 3 we show that despite its non-convexity, the optimal solutions can be very well characterized. We prove that the optimal rate allocations are monotonic in a function of the path loss. Based on this property, we show that in the optimal rate allocation, only 3 rates are given to users. In Section 4 we propose a polynomial time algorithm in the number of users that solves optimally the joint rate and power allocation problem. Our algorithm can be generalized to solve the optimal rate/power allocation problem in small networks, thus providing a first step into the direction of fast algorithms for resource allocation in a large network. We conclude with some remarks and open problems in Section 5.

\section{$2 \quad$ Model}

We consider a system with mobile users served by 2 base transmitter stations (BTSs), X and Y. Denote by $U_{X}$, respectively $U_{Y}$, the set of mobiles served by BTS X, respectively BTS Y. Let $l_{i, X}$ denote the path loss from BTS $X$ to mobile $i$, let $N_{i}$ be the thermal noise at the location of mobile $i$, and let $\epsilon_{i}$ denote the energy per bit to interference ratio requirement for mobile $i$. Let $P_{i X}$ denote the transmission power of BTS $X$ to mobile $i$, and $P_{X}$ the maximum down link transmission power of BTS $X$. The power received by mobile $i$ from BTS $X$ is $P_{i X}^{r e c}=P_{i X} l_{i, X}$. We assume that mobiles are served by a single BTS, which is 
a natural assumption for moving mobiles. A configuration of mobiles is feasible when for each mobile $i$ served by BTS $X$, say, the energy per bit to interference ratio exceeds the threshold $\epsilon_{i}$. If a configuration is feasible, then under perfect power control the energy per bit to interference ratio $\left(\frac{E_{b}}{I_{0}}\right)_{i}$ equals this threshold. Thus, assuming perfect power control, feasibility for a configuration in which mobile $i$ is served by BTS $X$ is characterized by,

$$
\left(\frac{E_{b}}{I_{0}}\right)_{i}:=\frac{W}{r_{i}} \frac{P_{i X} l_{i, X}}{\alpha l_{i, X}\left(\sum_{j \in U_{X}} P_{j X}-P_{i X}\right)+l_{i, Y} \sum_{j \in U_{Y}} P_{j Y}+N_{i}}=\epsilon_{i},
$$

where $U_{X}$ is the set of mobiles served by BTS $X, W$ is the system chip rate, $\alpha$ is the down link orthogonality factor, and $r_{i}$ is the data rate for mobile $i$.

Data rates can be assigned from the continuous interval $\left[r_{\min }, R_{\max }\right]$, with $r_{\min }>0$. The optimization problem is to determine an assignment of rates and powers to mobiles that maximizes the total rate.

For each fixed number of mobile calls placed in the coverage area, the rate assignment problem can be formulated as the following optimization problem:

$$
\begin{aligned}
\max & \sum_{i \in U} r_{i} \\
\text { s.t. } & \left(\frac{E_{b}}{I_{0}}\right)_{i}=\epsilon_{i}, \quad i \in U, \\
\mathrm{P}(\mathrm{n}) \quad & \sum_{i \in U_{X}} P_{i X} \leq P_{X}, \\
& \sum_{i \in U_{Y}} P_{i Y} \leq P_{Y}, \\
& r_{i} \in\left[r_{\min }, R_{\max }\right], \quad i \in U, \\
& P_{i X} \geq 0, \quad \forall i \in U_{X} \cup U_{Y} .
\end{aligned}
$$

\section{Characterization of an Optimal Rate Assignment}

For clarity of presentation, we assume that all users have the same threshold $\epsilon_{i}=\epsilon$. Denote $V\left(r_{i}\right)=\frac{\epsilon r_{i}}{W+\alpha \epsilon r_{i}}$, and let

$$
l_{i}=\left\{\begin{array}{l}
\frac{l_{i, Y}}{l_{i, X}}, \text { for } i \in U_{X} \\
\frac{l_{i, X}}{l_{i, Y}}, \text { for } i \in U_{Y}
\end{array}\right.
$$

According to Lemma 1.1. in [4], $P(n)$ can be rewritten as: 


$$
\begin{aligned}
P(n) & : R(n)=\max \sum_{i \in U} r_{i} \\
& \text { s.t. }\left(1-\alpha \sum_{i \in U_{X}} V\left(r_{i}\right)\right) x-\sum_{i \in U_{X}} V\left(r_{i}\right) l_{i} y-\sum_{i \in U_{X}} V\left(r_{i}\right) l_{i, X}^{-1} N_{i}=0, \\
& -\sum_{i \in U_{Y}} V\left(r_{i}\right) l_{i} x+\left(1-\alpha \sum_{i \in U_{Y}} V\left(r_{i}\right)\right) y-\sum_{i \in U_{Y}} V\left(r_{i}\right) l_{i, Y}^{-1} N_{i}^{i}=0 \\
& P_{X}-x \geq 0, \\
& P_{Y}-y \geq 0, \\
& x \geq 0, \\
& y \geq 0, \\
& R_{\max }-r_{i} \geq 0, \text { for } i \in U_{X} \cup U_{Y} \\
& r_{i}-r_{\min } \geq 0, \text { for } i \in U_{X} \cup U_{Y} .
\end{aligned}
$$

Notice that this is neither a linear programming nor a convex programming problem. We assume that the rate assignment problem above has at least one feasible solution, or, in other words, that there exist powers $P_{X}, P_{Y}$, such that assigning minimum rate to all users is feasible.

For later reference, we also provide the Lagrangian. Let $\lambda \in \mathbb{R}^{6}, \mu, \nu \in \mathbb{R}^{|U|}$ be the Lagrangian multipliers corresponding to equations (2)-(9). Denote by $r=\left(r_{i}\right)_{i \in U_{X} \cup U_{Y}}$ the vector of the rates allocated to users. The Lagrangian corresponding to $P(n)$ is

$$
\begin{aligned}
L(x, y, r, \lambda, \mu, \nu) & =\sum_{i \in U} r_{i} \\
& \left.+\lambda_{1}\left(\left(1-\alpha \sum_{i \in U_{X}} V\left(r_{i}\right)\right) x-\sum_{i \in U_{X}} V\left(r_{i}\right) l_{i} y-\sum_{i \in U_{X}} V\left(r_{i}\right) l_{i, X^{-1}}^{-1} N_{i}\right)\right) \\
& +\lambda_{2}\left(-\sum_{i \in U_{Y}} V\left(r_{i}\right) l_{i} x+\left(1-\alpha \sum_{i \in U_{Y}} V\left(r_{i}\right)\right) y-\sum_{i \in U_{Y}} V\left(r_{i} l_{i, Y}^{-1} N_{0}\right)\right. \\
& +\lambda_{3}\left(P_{X}-x\right)+\lambda_{4}\left(P_{Y}-y\right)+\lambda_{5} x+\lambda_{6} y \\
& +\sum_{i \in U} \mu_{i}\left(R_{\max }-r_{i}\right)+\sum_{i \in U} \nu_{i}\left(r_{i}-r_{\min }\right) .
\end{aligned}
$$

Next we will characterize the optimal rate assignment. We start with a monotonicity property of the rates.

Theorem 1. If $P(n)$ is feasible, and $\left(x^{*}, y^{*}, r^{*}\right)$ is an optimal solution, then for any two calls $i$ and $j$, say, in cell $X$,

$$
y^{*} l_{i}+l_{i, X}^{-1} N_{i}<y^{*} l_{j}+l_{j, X}^{-1} N_{j} \Rightarrow r_{i}^{*} \geq r_{j}^{*} .
$$

A similar statement holds for cell $Y$. 
Proof. Suppose there exist two calls $i, j \in U_{X}$ such that $l_{i} y^{*}+l_{i, X}^{-1} N_{i}<l_{j} y^{*}+$ $l_{j, X}^{-1} N_{j}$ and $r_{i}^{*}<r_{j}^{*}$.

Define the following rate vector $\hat{r} \in \mathbb{R}^{\left|U_{X}\right|+\left|U_{Y}\right|}$ :

$$
\hat{r_{k}}=\left\{\begin{array}{l}
r_{k}^{*}, \text { for } k \in U_{X} \cup U_{Y} \backslash\{i, j\} \\
r_{j}^{*}, \text { for } k=i, \\
r_{i}^{*}, \text { for } k=j,
\end{array}\right.
$$

i.e., with rate assignment to calls $i$ and $j$ interchanged. As the total rate is unchanged, the throughput of the rate assignments $r$ and $\hat{r}$ is the same. Let

$$
\hat{x}=\frac{\sum_{i \in U_{X}} V\left(\hat{r}_{i}\right)\left(l_{i} y^{*}+l_{i, X}^{-1} N_{i}\right)}{1-\alpha \sum_{i \in U_{X}} V\left(\hat{r}_{i}\right)} .
$$

It can be easily seen that $\hat{x}<x^{*}$.

Note that $\left(\hat{x}, y^{*}, \hat{r}\right)$ is not a feasible solution of $P(n)$, since it does not satisfy constraints (2) and (3). However, we can obtain a feasible solution by increasing the rates $\hat{r}$ for users in $U_{X} \backslash\{j\}$, until power $x^{*}$ is reached in (11) or all rates in $U_{X} \backslash\{j\}$ are maximal. Denote by $(\tilde{r})_{U_{X}}$ the rate assignment obtained in this way. Suppose that $\left(\tilde{r_{k}}\right)_{k \in U_{X} \backslash\{j\}}=\left(R_{\max }\right)_{U_{X} \backslash\{j\}}$. By decreasing $y^{*}$ such that (3) is satisfied, while the rates for users in $U_{Y}$ remain the same, we obtain a power/rate allocation with a higher throughput then $r^{*}$. If $x^{*}$ was reached in (11), then $\left(x^{*}, y^{*},\left(\tilde{r_{k}}\right)_{k \in U_{X}},\left(r_{k}^{*}\right)_{k \in U_{Y}}\right)$ is a feasible solution of $P(n)$ which gives a higher throughput then $\left(x^{*}, y^{*},\left(\left(r_{k}^{*}\right)_{k \in U_{X}},\left(r_{k}^{*}\right)_{k \in U_{Y}}\right)\right.$. This contradicts the fact that $\left(x^{*}, y^{*}, r^{*}\right)$ is an optimal solution. Hence, it must be that $r_{i}^{*} \geq r_{j}^{*}$.

Denote by $h_{1}(x, y, r), \ldots, h_{6}(x, y, r)$ the functions in the left hand side of constraints (2)-(17) and by $g_{1}(x, y, r), \ldots, g_{2\left|U_{X}\right|+2\left|U_{Y}\right|}(x, y, r)$ the functions in the left hand side of constraints (8)-(9).

We will first review some optimization terminology (see [3]). If an inequality constraint of $P(n)$ is satisfied with equality in a feasible vector $(x, y, r) \in$ $\mathbb{R}^{\left|U_{X}\right|+\left|U_{Y}\right|+2}$ of $P(\mathbf{n})$, the constraint is active in $(x, y, r)$. Denote by $A(x, y, r)$ the set of active inequalities in the point $(x, y, r)$. A feasible vector $(x, y, r)$ is regular if the gradients $\nabla h_{1}(x, y, r), \nabla h_{2}(x, y, r)$ and $\nabla h_{i}(x, y, r), \nabla g_{j}(x, y, r)$ for $i \in A(x, y, r) \bigcap\{3,4,5,6\}, j \in A(x, y, r)$ are linearly independent.

Notice that $\nabla h_{1}(x, y, r), \nabla h_{2}(x, y, r)$ are linearly independent for any feasible $(x, y, r)$, so that all points with $A(x, y, r)=\emptyset$ are regular. Further, note that since $r_{\min }>0, x \neq 0$ and $y \neq 0$ in the optimal solution. Moreover, since the objective function is linear, each optimum must be a global optimum.

We will start by characterizing the rate assignment for regular points. In the proofs that follow, we will make use of the Karush-Kuhn-Tucker (KKT) necessary conditions for a regular point to be an optimal solution (see [3]). They state that for a regular point $\left(x^{*}, y^{*}, r^{*}\right)$ that is an optimum of $P(n)$ there exists an unique multiplier vector $\left(\lambda^{*}, \mu^{*}, \nu^{*}\right)$ such that:

(K1) $\nabla\left(x^{*}, y^{*}, r^{*}\right) L\left(x^{*}, y^{*}, r_{i}^{*}, \lambda^{*}, \mu^{*}, \nu^{*}\right)=0$, where $L$ denotes the Lagrangian function corresponding to $P(\mathbf{n})$. 
(K2) $\lambda_{k}^{*} \geq 0$, for $k \in\{3,4,5,6\}, \mu^{*} \geq 0$ and $\nu^{*} \geq 0$,

(K3) The Lagrangian multipliers corresponding to non active constraints are equal to 0 .

Theorem 2. If $P(n)$ is feasible and $\left(x^{*}, y^{*}, r^{*}\right)$ a regular optimal solution, then a) $x^{*}=P_{X}$ or $y^{*}=P_{Y}$ or $r_{i}^{*}=R_{\max }$, for each call $i \in U_{X} \cup U_{Y}$.

b) If the rates of two calls $i, j \in U_{X}$ satisfy $r_{\min }<r_{i}<R_{\max }$ and $r_{\min }<r_{j}<$ $R_{\max }$, then $l_{i} y^{*}+l_{i, X}^{-1} N_{i}=l_{j} y^{*}+l_{j, X}^{-1} N_{j}$ and $r_{i}=r_{j}$. A similar statement holds for cell $Y$.

Proof. a) Note that since the minimum rate can be ensured to all accepted users, constraints (2) and (3) imply that $x^{*}>0$ and $y^{*}>0$. Thus, based on condition (K3), we conclude that $\lambda_{5}^{*}=\lambda_{6}^{*}=0$. Suppose that $x^{*}<P_{X}, y<P_{Y}$ and $r_{\min } \leq r_{i}<R_{\max }$ for a call $i \in U_{X}$, say.

From (K3), follows that $\lambda_{3}^{*}=\lambda_{4}^{*}=0$ and that $\mu_{i}^{*}=0$.

Moreover, (K1) imply that $\frac{\partial L}{\partial x}\left(x^{*}, y^{*}, r^{*}, \lambda^{*}, \mu^{*}, \nu^{*}\right)=0, \frac{\partial L}{\partial y}\left(x^{*}, y^{*}, r_{i}^{*}, \lambda^{*}, \mu^{*}, \nu^{*}\right)$ $=0$, and $\frac{\partial L}{\partial r_{i}}\left(x^{*}, y^{*}, r^{*}, \lambda^{*}, \mu^{*}, \nu^{*}\right)=0$. Hence,

$$
\begin{cases}\lambda_{1}^{*}\left(1-\alpha \sum_{i \in U_{X}} V\left(r_{i}^{*}\right)\right)-\lambda_{2}^{*} \sum_{i \in U_{Y}} V\left(r_{i}^{*}\right) l_{i} & =0 \\ -\lambda_{1}^{*} \sum_{i \in U_{X}} V\left(r_{i}^{*}\right) l_{i}+\lambda_{2}^{*}\left(1-\alpha \sum_{i \in U_{Y}} V\left(r_{i}^{*}\right)\right) & =0 . \\ 1+\nu_{i}^{*}-\mu_{i}^{*}-\lambda_{1}^{*} V^{\prime}\left(r_{i *}^{*}\right)\left(\alpha x^{*}+l_{i} y^{*}+l_{i, X}^{-1} N_{i}\right)=0 . & \end{cases}
$$

Observe that the first two equations in $\lambda_{1}^{*}, \lambda_{2}^{*}$ are linearly independent (recall constraints (2)-(3) and the assumption that a minimal rate assignment is feasible), so the only solution is $\lambda_{1}^{*}=\lambda_{2}^{*}=0$.

Further, since $\mu_{i}^{*}=0$, from the third equation in (12) follows that $\nu_{i}=-1$, which contradicts condition (K2), that $\nu_{i}^{*} \geq 0$.

Hence, in an optimal solution, either the rates of all users are maximal, or the power in one of the cells is maximal.

b) Suppose that there exist two different values $l_{i} y^{*}+l_{i, X}^{-1} N_{i}, l_{j} y^{*}+l_{j, X}^{-1} N_{j}$, respectively, for which the corresponding rates are $r_{\min }<r_{i}^{*}<R_{\max }$ and $r_{\min }<$ $r_{j}^{*}<R_{\max }$. Without loss of generality, we assume that $l_{i} y^{*}+l_{i, X}^{-1} N_{i}<l_{j} y^{*}+$ $l_{j, X}^{-1} N_{j}$. From Theorem 1 it follows that $r_{i}^{*} \geq r_{j}^{*}$.

Since $r_{\min }<r_{i}^{*}<R_{\max }$ and $r_{\min }<r_{j}^{*}<R_{\max }$, condition (K3) imply that

$$
\mu_{i}=\mu_{j}=\nu_{i}=\nu_{j}=0 .
$$

Hence, (12) implies that

$$
\frac{V^{\prime}\left(r_{i}^{*}\right)}{V^{\prime}\left(r_{j}^{*}\right)}=\frac{\alpha x^{*}+l_{j} y^{*}+l_{j, X}^{-1} N_{j}}{\alpha x^{*}+l_{i} y^{*}+l_{i, X}^{-1} N_{i}} .
$$

Our assumption $l_{j} y^{*}+l_{j, X}^{-1} N_{j}>l_{i} y^{*}+l_{i, X}^{-1} N_{i}$ implies that $V^{\prime}\left(r_{i}^{*}\right)>V^{\prime}\left(r_{j}^{*}\right)$. Since the function $V^{\prime}$ is decreasing, it follows that $r_{i}^{*}<r_{j}^{*}$, which contradicts Theorem 
11. We conclude that if the rates of two users $i, j \in U_{X}$ satisfy $r_{\min }<r_{i}^{*}<R_{\max }$ and $r_{\min }<r_{j}^{*}<R_{\max }$, then $l_{i} y^{*}+l_{i, X}^{-1} N_{i}=l_{j} y^{*}+l_{j, X}^{-1} N_{j}$. Clearly, it then follows that $r_{i}^{*}=r_{j}^{*}$.

Corollary 1. Let $\left(x^{*}, y^{*}, r^{*}\right)$ be regular and an optimal solution of problem $P(n)$. Suppose that calls in cell $X$, respectively in cell $Y$ are ordered in increasing order of their $l_{i} y^{*}+l_{i, X}^{-1} N_{i}$, respectively $l_{j} x^{*}+l_{j, Y}^{-1} N_{j}$ values. Then, there exists a positive number $A\left(y^{*}\right)$, such that for each $i \in U_{X}$ with $l_{i} y^{*}+l_{i, X}^{-1} N_{i}<A\left(y^{*}\right)$, $r_{i}^{*}=R_{\max }$ and for each $i \in U_{X}$ with $l_{i} y^{*}+l_{i, X}^{-1} N_{i}>A\left(y^{*}\right), r_{i}^{*}=r_{\min }$. Moreover, there exists a positive number $B\left(x^{*}\right)$, such that for each $j \in U_{Y}$ with $l_{j} x^{*}+l_{j, Y}^{-1} N_{j}<B\left(x^{*}\right), r_{j}^{*}=R_{\max }$ and for each $j \in U_{Y}$ with $l_{j} x^{*}+l_{j, Y}^{-1} N_{j}>$ $B\left(x^{*}\right), r_{j}^{*}=r_{\min }$.

For a non regular point, the following theorem gives a complete characterization of the optimal power and rate assignment.

Theorem 3. For each non regular point $(x, y, r)$, the following conditions are satisfied:

a) $x=P_{X}$ or $y=P_{Y}$

b) If $x=P_{X}$ and $y \neq P_{Y}$, then $r_{i} \in\left\{r_{\min }, R_{\max }\right\}$, for each $i \in U_{X}$.

c) If $y=P_{Y}$ and $x \neq P_{X}$, then $r_{i} \in\left\{r_{\min }, R_{\max }\right\}$, for each $i \in U_{Y}$.

Proof. Let $(x, y, r)$ be a non regular point, feasible for $P(\mathbf{n})$. Consider the matrix $M$ formed by the $\nabla h_{1}(x, y, r), \nabla h_{2}(x, y, r)$ and $\nabla h_{i}(x, y, r), \nabla g_{j}(x, y, r)$ for $i \in$ $A(x, y, r) \bigcap\{3,4,5,6\}, j \in A(x, y, r)$. Let $K$ be the number of active inequality constraints. Notice that for a non-regular point it must be that $K>0$, since $\nabla h_{1}(x, y, r), \nabla h_{2}(x, y, r)$ are linearly independent. Clearly, $2 \leq \operatorname{rank}(M) \leq$ $K+2$.

a) Suppose that $x \neq P_{X}$ and that $y \neq P_{Y}$. In other words, the active inequality constraints correspond to the constraints on rates. Then, matrix $M$ has the following form:

$$
M=\left(\begin{array}{cccc}
1-\alpha \sum_{i \in U_{X}} V\left(r_{i}\right) & -\sum_{i \in U_{X}} V\left(r_{i}\right) l_{i} & A & \mathbf{0} \\
-\sum_{i \in U_{Y}} V\left(r_{i}\right) l_{i} & 1-\alpha \sum_{i \in U_{Y}} V\left(r_{i}\right) & \mathbf{0} & B \\
0 & 0 & C & \mathbf{0} \\
0 & 0 & \mathbf{0} & D
\end{array}\right)
$$

where the vectors $A \in \mathbb{R}^{\left|U_{X}\right|}, B \in \mathbb{R}^{\left|U_{Y}\right|}$ are defined as follows:

$$
A=\left[-V^{\prime}\left(r_{i}\right)\left(\alpha x+l_{i} y+l_{i, X^{-1}}^{-1} N_{i}\right)\right]_{i \in U_{X}}, \quad B=\left[-V^{\prime}\left(r_{i}\right)\left(\alpha y+l_{i} x+l_{i, Y}^{-1} N_{i}\right)\right]_{i \in U_{Y}},
$$

and the matrices $C \in \mathbb{R}^{\left|\left\{i \in U_{X}: g_{i} \in A(x, y, r)\right\}\right|} \times \mathbb{R}^{\left|\left\{i \in U_{X}\right\}\right|}, D \in \mathbb{R}^{\left|\left\{i \in U_{Y}: g_{i} \in A(x, y, r)\right\}\right|}$ $\times \mathbb{R}^{\left|\left\{i \in U_{Y}\right\}\right|}$ are obtained from the diagonal square matrices with diagonal $\left.\left.\operatorname{diag}(\bar{C})=\left[I_{\left\{r_{i}=r_{\min }\right\}}\right)-I_{\left\{r_{i}=R_{\max }\right\}}\right]_{\left\{i \in U_{X}\right\}}, \operatorname{diag}(\bar{D})=\left[I_{\left\{r_{i}=r_{\min }\right\}}\right)-I_{\left\{r_{i}=R_{\max }\right\}}\right]_{\left\{i \in U_{Y}\right\}}$, 
by deleting all rows for which the diagonal elements equals zero, where $I_{\{a\}}=1$ if $a$ is true, and 0 otherwise.

Clearly, $\operatorname{rank}(C)+\operatorname{rank}(D)=K$. Since constraints $\nabla h_{1}(x, y, r), \nabla h_{2}(x, y, r)$ are linearly independent, it follows that $\operatorname{rank}(M)=K+2$, which contradicts the fact that $(x, y, r)$ is non regular. Hence, in a non regular point, the power assigned to one of the cells has to be maximal.

b) Suppose that $x=P_{X}$ and $y \neq P_{Y}$ and that there exist $i \in U_{X}$ such that $r_{\min }<r_{i}<R_{\max }$. It can be proved that the rank of the matrix $M$ is again $\operatorname{rank}(M)=K+2$, which contradicts the fact that $(x, y, r)$ is non regular.

c) The proof is similar to b).

\section{Algorithm for Optimal Rate and Power Assignment}

Based on Theorems 13 and Corollary 1, we now propose on algorithm for finding the optimal solution of $P(n)$. The algorithm relies on a reduction of the optimization problem $P(n)$ to a series of optimization problems in $\mathbb{R}$. Notice that the algorithm considers the regular and non regular points.

If maximum rate to all users is feasible, then the optimal solution has been found. To check whether the maximum rate is feasible, one only has to check if the corresponding powers calculated from (2)-(3) satisfy $0 \leq x \leq P_{X}$ and $0 \leq y \leq P_{Y}$. If this is not the case, then the algorithm calculates the rate allocation achieving maximum throughput for the case when the power in cell $\mathrm{X}$ is maximal, respectively the power in cell $\mathrm{Y}$ is maximal. The algorithm will choose among these 2 allocations the one with higher throughput. Note that if the rates are known, from (2), (3) and (1) the powers of each user can be derived.

Next we will consider the case when in cell $\mathrm{X}$ the base station transmits at maximum power, i.e., $x^{*}=P_{X}$. The case $y^{*}=P_{Y}$ can be treated similarly. The algorithm provides a reduction of the optimization problem $P(n)$ that is based on a search procedure to find the values $B\left(x^{*}\right)$ and $A\left(y^{*}\right)$ introduced in Corollary 1 to obtain the set of mobiles at which the rate drops from $R_{\max }$ to $r_{\min }$ in both cells. As the set of mobiles for maximum power at cell $X$ also depends on the power assignment in cell $Y$, these sets cannot be determined independently.

Order the locations in cell $\mathrm{Y}$ in increasing order of $l_{j} P_{X}+l_{j, Y}^{-1} N_{j}$.

According to Theorem $2 \mathrm{~b})$ all users $j$ in cell $\mathrm{Y}$ with rate $r_{j} \in\left(r_{\min }, R_{\max }\right)$ are characterized by the same value of $l_{j} P_{X}+l_{j, Y}^{-1} N_{j}$ and have the same rate $r_{X}$. Let $B\left(P_{X}\right)$ be this value and $U_{Y}\left(B\left(P_{X}\right)\right)=\left\{j \in U_{Y}: l_{j} P_{X}+l_{j, Y}^{-1} N_{j}=B\left(P_{X}\right)\right\}$. From Theorem 1 and Theorem 2 follows that for each $j \in U_{Y}$ with $l_{j} P_{X}+$ $l_{j, Y}^{-1} N_{j}<B\left(P_{X}\right), r_{j}^{*}=R_{\max }$ and for each $j \in U_{Y}$ with $l_{j} P_{X}+l_{j, Y}^{-1} N_{j}>B\left(P_{X}\right)$, $r_{j}^{*}=r_{\min }$. Suppose that $s$ users in $U_{Y}\left(B\left(P_{X}\right)\right)$ have rate $R_{\max }, v$ users have rate $r_{\min }$ and the rest have rate $r_{Y}$. The rate $r_{Y}$ is unknown at this stage of the algorithm. The power assigned to cell $Y$, as a function of $r_{Y}$, can be determined from constraint (3), and is given by 


$$
y^{*}\left(r_{Y}\right)=\frac{\sum_{j \in U_{Y} \backslash U_{Y}\left(B\left(P_{X}\right)\right)} V\left(r_{j}\right)\left(l_{j} P_{X}+l_{j, X}^{-1} N_{j}\right)+\left(s V\left(R_{\max }\right)+v V\left(r_{\min }\right)+t V\left(r_{Y}\right)\right) B\left(P_{X}\right)}{\left.1-\alpha\left(\sum_{j \in U_{Y} \backslash U_{Y}\left(B\left(P_{X}\right)\right)} V\left(r_{j}\right)+s V\left(R_{\max }\right)+v V\left(r_{\min }\right)\right)+t V\left(r_{Y}\right)\right)} .
$$

Similarly, for a specific $y^{*}\left(r_{Y}\right)$, Theorem 2b), implies that all the users $i$ in cell $\mathrm{X}$ with $r_{i} \in\left(r_{\min }, R_{\max }\right)$ are characterized by the same value of $l_{i} y^{*}\left(r_{Y}\right)+l_{i, Y}^{-1} N_{0}^{i}$, say $A\left(y^{*}\left(r_{Y}\right)\right)$. Denote by $U_{X}(A, B)=\left\{i \in U_{X}: l_{i} y^{*}\left(r_{Y}\right)+l_{i, X}^{-1} N_{i}=A\left(y^{*}\left(r_{Y}\right)\right)\right\}$. Then all $i \in U_{X}$ with $l_{i} y^{*}\left(r_{Y}\right)+l_{i, X}^{-1} N_{i}<A\left(y^{*}\left(r_{Y}\right)\right)$, have rate $R_{\max }$ and all $i \in U_{X}$ with $l_{i} y^{*}\left(r_{Y}\right)+l_{i, X}^{-1} N_{i}>A\left(y^{*}\left(r_{Y}\right)\right)$ have rate $r_{\min }$. Suppose that $u$ users in $U_{X}(A, B)$ have rate $R_{\max }, z$ users have a rate $r_{X} \in\left(r_{\min }, R_{\max }\right)$ and the rest have rate $r_{\min }$. Then the rate $r_{X}$ can be expressed from (2) as follows:

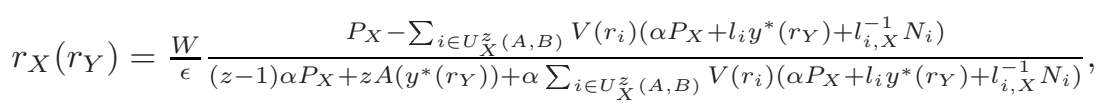

where $U_{X}^{z}(A, B)$ denotes the set of users in $U_{X}$ with rate $r \in\left(r_{\min }, R_{\max }\right)$.

Note that if $B\left(P_{X}\right), s, v, u, z$ were known, $r_{Y}$ would be the only unknown. This suggests that by enumerating all the possible values of $B\left(P_{X}\right), s, v, u, z$, the problem could be reduced to an optimization problem in one variable, $r_{Y}$. The optimization problem is not easy to formulate due to the fact that the value of $r_{Y}$, more precisely $y^{*}\left(r_{Y}\right)$, is a decision variable in the assignment of $R_{\max }$ and $r_{\min }$ to users in $U_{X}$ (see Corollary 3 ). However, it can be easily seen that only some values of $y^{*}\left(r_{Y}\right)$ induce a different rate allocation in cell X. Let

$$
L=\left\{\frac{l_{j_{1, X}}^{-1} N_{j_{1}}-l_{j_{2}, X}^{-1} N_{j_{2}}}{l_{j_{2}}-l_{j_{1}}}, j_{1}, j_{2} \in U_{X}\right\} \bigcap R^{+} .
$$

Suppose that $L \neq \emptyset$. For all $y^{*}\left(r_{Y}\right) \in\left[L_{i}, L_{i+1}\right)$ the ordering of mobiles in cell $\mathrm{X}$, as determined by their value of $l_{i} y^{*}\left(r_{Y}\right)+l_{i, X}^{-1} N_{i}$ is the same, but for different intervals $\left[L_{j}, L_{j+1}\right)$ this ordering may be different. Note that $V(r)$ is strictly increasing, so that $y^{*}\left(r_{Y}\right)$ is strictly increasing. As a consequence, each unknown $y^{*}\left(r_{Y}\right) \in\left[L_{i}, L_{i+1}\right)$ yields a unique $r_{Y}$.

Hence, for $y^{*}\left(r_{Y}\right) \in\left[L_{i}, L_{i+1}\right), P(n)$ can be reduced to the following optimization problem in $\mathbb{R}$ :

$$
\begin{aligned}
\max \quad z r_{X}\left(r_{Y}\right) & +\operatorname{tr}_{Y} \\
\text { s.t. } \quad y^{*}\left(r_{Y}\right) & \leq P_{Y} \\
y^{*}\left(r_{Y}\right) & \in\left[L_{i}, L_{i+1}\right] \\
r_{X}\left(r_{Y}\right) & \in\left[r_{\min }, R_{\max }\right] \\
r_{Y} & \in\left[r_{\min }, R_{\max }\right] .
\end{aligned}
$$

Thus, the original rate optimization problem can be reduced to $O\left(\left|U_{X}\right|^{2}\right)$ optimization problems in $\mathbb{R}$, one for each interval $\left[L_{i}, L_{i+1}\right)$.

If $L=\emptyset$, then the order of the users in $U_{X}$ does not depend on $y^{*}\left(r_{Y}\right)$ and we obtain a similar optimization problem to (13), without the second constraint.

Note that the optimization problems (13) are constraint optimization problems in one variable, which can be easily solved. 


\section{Conclusions}

In this paper we have proposed an exact algorithm for the joint rate and power allocation problem in two cells of a CDMA network. We have analyzed several properties of the optimal solutions, based on which, we have proposed a polynomial time algorithm for solving the problem. Our results can be extended to non-decreasing utility functions at the cost of a rather involved notation. Moreover, the algorithm can be extended to iteratively solve the rate/power allocation problem in a small number of cells.

\section{References}

1. J.B. Andersen, T.S. Rappaport and S. Yoshida, Propagation measurements and models for wireless communications channels, IEEE Commun. Mag., vol. 33, pp. 42-49, 1995

2. F. Berggren, Distributed power control for throughput balancing in CDMA systems, in Proceedings of IEEE PIMRC, vol. 1, pp. 24-28, 2001.

3. D. Bertsekas, Nonlinear programming, Athena scientific, 2003

4. R.J. Boucherie, A. Bumb, A.I. Endrayanto, G.J. Woeginger, A combinatorial approximation algorithm for CDMA downlink rate allocation, in: Tellecomunications Planning : Innovations in pricing, network design and management, S. Raghavan, G. Anandalingam eds., Springer, pp. 175-193, 2006.

5. X. Duan, Z. Niu, J. Zheng, Downlink Transmit Power Minimization in PowerControlled Multimedia CDMA Systems, in Proceedings of IEEE 13th Int. Symposium Personal, Indoor and Mobile Radio Communication, 2002.

6. A.I. Endrayanto, J.L van den Berg, R.J Boucherie, An analytical model for CDMA downlink rate optimization taking into account uplink coverage restrictions, Performance Evaluation, Vol 59. pp. 225-246, 2005.

7. S. V. Hanly. An algorithm of combined cell-site selection and power control to maximize cellular spread spectrum capacity, IEEE Journal on Selected Areas in Communications, Vol. 13 no. 7 pp. 1332-1340, 1995.

8. J.-W. Lee, R.R. Mazumdar and N.B. Shroff, Downlink power allocation for multiclass wireless systems. in IEEE/ACM Trans. Netw., vol. 13, pp.854-867, 2005.

9. J.-W. Lee, R.R. Mazumdar and N.B. Shroff, Joint resource allocation and base station assignment for the downlink in CDMA networks, IEEE/ACM Trans. Netw., vol. 14, no. 1, pp.1-14, 2006.

10. D. O 'Neill, D. Julian and D. Boyd, Seeking Foschini's Genie: Optimal Rates and Powers in Wireless Networks, to appear in IEEE Transactions on Vehicular Technology.

11. R. D. Yates, A Framework for Uplink Power Control in Cellular Radio Systems, IEEE Journal of Selected Areas in Communications, Vol. 13, no. 7, pp. 1341-1347, 1995.

12. Z. Yin and J.Xie, Joint power and rate allocation for the downlink in wireless CDMA networks, The 14th IEEE International Symposium on Personal, Indoor and Mobile Communication Proceedings, pp.326-330, 2003 